\title{
Optical fiber based ammonia gas sensor with carbon nanotubes sensing enhancement
}

\begin{abstract}
In this research, we develop an ammonia gas sensor using plastic optical fibers. These chemically etched optical fibers were layered with a thin film of carbon nanotubes to enhance the sensing capability of the developed sensor. The sensor was then interrogated with different ratios of gaseous ammonia to synthetic air using a controlled gas testing setup. The developed sensor exhibited high sensitivity of $12 / \mathrm{Vol} \%$ of ammonia when tested with to various ratios of ammonia to air. The high response and recovery time of the sensor was calculated to be 25 and 23 seconds respectively makes it a promising contender in gas sensing applications.
\end{abstract}

Keyword: Fiber optic; Sensor; Ammonia carbon nanotubes; Gas sensor 